\title{
The effects of Si-doped prelayers on the optical properties of InGaN/GaN single quantum well structures.
}

\author{
M. J. Davies, ${ }^{1,}$ a) P. Dawson, ${ }^{1}$ F. C.-P. Massabuau, ${ }^{2}$ R. A. Oliver, ${ }^{2}$ M. J. Kappers, ${ }^{2}$ and C. J. Humphreys ${ }^{2}$ \\ ${ }^{1)}$ School of Physics and Astronomy, Photon Science Institute, University of Manchester, Manchester, UK, \\ M13 9PL. \\ ${ }^{2)}$ Department of Material Science and Metallurgy, 27 Charles Babbage Road, University of Cambridge, Cambridge, \\ UK, CB3 OFS.
}

(Dated: 13 August 2014)

\begin{abstract}
In this paper we report on the effects of including Si-doped (In)GaN prelayers on the low temperature optical properties of a blue-light emitting InGaN/GaN single quantum well. We observed a large blue shift of the photoluminescence peak emission energy and significant increases in the radiative recombination rate for the quantum well structures that incorporated Si-doped prelayers. Simulations of the variation of the conduction and valence band energies show that a strong modification of the band profile occurs for the quantum wells on Si-doped prelayers due to an increase in strength of the surface polarization field. The enhanced surface polarization field opposes the built-in field across the quantum well and thus reduces this built-in electric field. This reduction of the electric field across the quantum well reduces the Quantum Confined Stark Effect and is responsible for the observed blue shift and the change in the recombination dynamics.
\end{abstract}

InGaN/GaN quantum wells (QWs) are frequently used as the active region for light emitting diodes (LEDs) that cover the ultraviolet to green spectral region ${ }^{1-4}$. In particular LEDs, deposited in the $c$-plane orientation, that emit in the blue region of the spectrum can have internal quantum efficiencies of $50 \%$ or significantly greater ${ }^{5-7}$. This is despite the strong internal polarisation fields $s^{1,8,9}$ that exist in $c$-plane InGaN/GaN QW structures which lead to a large Quantum Confined Stark Effect (QCSE). The strong polarisation fields occur due to a combination of the spontaneous polarisation of the lattice and a strain-induced piezoelectric field due to the significant lattice mismatch between $\mathrm{GaN}$ and $\mathrm{InGaN}^{9}$. The large QCSE results in the spatial separation of the electron and hole wave functions leading to a reduction in the radiative recombination rate. Hence to maximise the radiative efficiency, steps must be taken to either reduce the non-radiative recombination rate or increase the radiative recombination rate. The radiative recombination rate would be increased if the internal strain, and hence the strength of the piezoelectric field in the InGaN QW, could be reduced. There are several reports that this can be achieved by the inclusion of a so-called InGaN prelayer prior to the growth of the QW stack. Typically a prelayer is a deliberately doped or undoped layer, $\sim 20 \mathrm{~nm}$ thick, with low In content and is positioned a few nm below the first QW. Alternatively, an InGaN/GaN superlattice can be used. Improvements in luminescence intensity and efficiencies have been reported for a range of structures and devices containing a variety of $n$-type doped InGaN prelayers ${ }^{2,3,10-12}$. In particular Nanhui et al. ${ }^{13,14}$ found that the PL intensity at room temperature from an InGaN/GaN multiple QW (MQW) structure increased with the inclusion of a

a) Matthew.Davies-2@Manchester.ac.uk
$20 \mathrm{~nm}$ thick $\operatorname{In}_{0.08} \mathrm{Ga}_{0.92} \mathrm{~N}$ prelayer, which was attributed to strain relaxation of the InGaN QWs. Otsuji et al. ${ }^{2}$ and Takahashi et al. ${ }^{10}$ attributed similar behaviour to an increase in electron capture efficiency, while Akasaka et $a l .^{11,12}$ and Törmä et al. ${ }^{3}$ ascribed the behaviour to a reduction in density of non-radiative recombination centres in the vicinity of the QWs. Takahashi et al. ${ }^{15}$ reported an increase in the formation of "V"-defects, which are believed to isolate threading dislocations from carriers within the $\mathrm{QWs}^{4}$. Recently we reported ${ }^{16}$ an improved room temperature internal quantum efficiency (IQE) for a 10 period $\operatorname{In}_{0.16} \mathrm{Ga}_{0.84} \mathrm{~N} / \mathrm{GaN} \mathrm{MQW}$ structure containing a $24 \mathrm{~nm}$ thick Si-doped $\mathrm{In}_{0.05} \mathrm{Ga}_{0.95} \mathrm{~N}$ prelayer. The increase in room temperature IQE was attributed to the contribution from an additional recombination process, on the high energy side of the spectrum, which had a faster radiative rate. The high energy emission was ascribed to either the recombination of carriers in QWs formed on semi-polar facets of the "V"-defects or in the $1^{\text {st }}$ QW whose properties had been modified by the prelayer. In summary, despite the reported improvements in luminescence properties due to the inclusion of InGaN prelayers no consensus has been reached for the precise role of the prelayer. As a logical extension to our previous work $^{16}$, we report in this paper on the effects of a range of Si-doped prelayers on the optical properties of single quantum well (SQW) InGaN/GaN structures.

The SQW structures studied were deposited by metalorganic vapor phase epitaxy on $c$-plane sapphire substrates (with a miscut of $0.25 \pm 0.1^{\circ}$ along $a$ ) in a $6 \times 2$ " Thomas Swan close-coupled showerhead reactor, using the "quasi-two temperature" growth method ${ }^{17}$. A range of $\mathrm{In}_{0.16} \mathrm{Ga}_{0.84} \mathrm{~N} / \mathrm{GaN}$ SQW structures were deposited on unintentionally-doped $\left(<10^{17} \mathrm{~cm}^{-3}\right)$ GaN templates, each containing different styles of prelayer prior to the growth of the SQW. The nominally $2.5 \mathrm{~nm}$-thick InGaN QW was capped with $7.5 \mathrm{~nm}$ of unintentionally doped GaN. To monitor the effects of the different prelayers on 
the optical properties of a SQW, a control sample (sample A) was grown which did not contain any prelayer or any intentionally doped layers. Sample B incorporated a $24 \mathrm{~nm}$ thick Si-doped $\left(5 \times 10^{18} \mathrm{~cm}^{3}\right) \mathrm{In}_{0.05} \mathrm{Ga}_{0.95} \mathrm{~N}$ layer, grown at a temperature of $770{ }^{\circ} \mathrm{C}, 3 \mathrm{~nm}$ below the $\mathrm{QW}$. The main characteristics of the prelayer in sample $\mathrm{B}$ that may influence the properties of the SQW are the presence of In (and indeed the indium content) and the fact that it was grown at a significantly lower temperature than the surrounding GaN. A reduced growth temperature might, for example, alter the formation of "V"-defects in the material underlying the QW, thus providing a mechanism for the effects of the prelayer. To monitor the effects of these two parameters Samples C and D were grown with $\mathrm{Si}$-doped $\left(5 \times 10^{18} \mathrm{~cm}^{3}\right) \mathrm{GaN}$ prelayers grown at $770^{\circ} \mathrm{C}$ and $1020^{\circ} \mathrm{C}$ respectively. Si-doping concentrations were based on secondary ion mass spectrometry analyses of calibration structures grown under similar conditions. The thickness and composition of the SQWs were determined by a combination of high resolution Xray diffraction (XRD) and X-ray reflectivity (XRR), as described by Smeeton et al. ${ }^{18}$. X-ray measurements were performed on a Philips X-pert Gen6 diffractometer. For XRD, a four-bounce asymmetric Ge (220) monochromator and a three-bounce Ge (220) analyser were employed. For each sample, a GaN (002) $\omega-2 \theta$ scan was used to determine the combined well and barrier thickness and the average indium content (i.e. average composition across the InGaN/GaN period). For XRR, a $4 \mathrm{~mm}$ mask was coupled with a $1 / 32^{\circ}$ programmable divergence slit and a $0.2 \mathrm{~mm}$ programmable receiving slit. The measured specular reflection of the X-ray beam contains a convolution of two Fabry-Pérot interference functions, occurring due to multiple reflections at the InGaN/GaN interfaces. The interplay between the two interference functions was used to determine the QW thickness following the method of Smeeton et al. ${ }^{18}$. The optical properties of the structures were studied at a temperature of $10 \mathrm{~K}$ by measuring the photoluminescence (PL) spectra and PL decay times. The samples were mounted at Brewsters angle on the cold finger of a variable temperature, closed-cycle helium cryostat to minimise the effects of Fabry-Pérot interference oscillations on the PL spectra ${ }^{19}$. For the PL spectroscopy a CW He/Cd laser, photon energy $3.815 \mathrm{eV}$, was used as the excitation source. The PL decay time measurements were performed using a frequency tripled, mode-locked, Ti:sapphire laser as the excitation source $(4.881 \mathrm{eV})$, with the signals being processed with a timecorrelated single photon counting system.

XRD and XRR characterisation performed on sample A and sample B showed that the QWs in the two structures are indistinguishable, with QW thicknesses of $2.3 \pm 0.1 \mathrm{~nm}$ and indium fractions of $15.7 \pm 1 \%$ and $16.2 \pm 1 \%$ respectively. Samples C and D were also measured to have to QW thicknesses of $2.4 \pm 0.1 \mathrm{~nm}$ and $2.3 \pm 0.1 \mathrm{~nm}$ with indium fractions of $16.0 \pm 1 \%$ and $16.2 \pm 1 \%$ respectively. As noted in the introduction it has been reported that $\mathrm{InGaN}$ prelayers may introduce

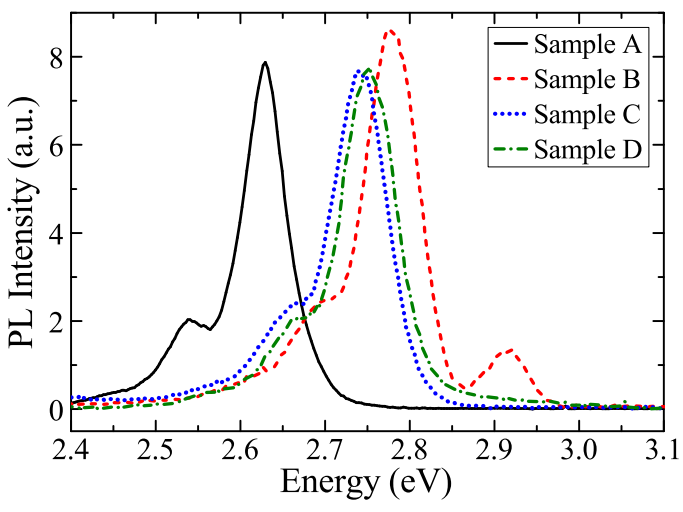

FIG. 1. PL spectra measured at low temperature $(10 \mathrm{~K})$ from samples A, B, C and D. Sample A is the reference structure, sample B contains the Si-doped InGaN prelayer and samples $\mathrm{C}$ and $\mathrm{D}$ contain the Si-doped GaN prelayers grown at 770 and $1020^{\circ} \mathrm{C}$ respectively.

strain relaxation. In single QW samples, the strain state cannot be reliably measured using an asymmetric reflection by XRD due to the low intensity of the peaks. In our previous work ${ }^{16}$ reciprocal space maps were recorded around the $\mathrm{GaN}$ (204) for 10-period $\mathrm{In}_{0.156} \mathrm{Ga}_{0.844} \mathrm{~N}(2.5$ $\mathrm{nm}) / \mathrm{GaN}(7.3 \mathrm{~nm})$ QW samples with and without a $24 \mathrm{~nm}$ thick Si-doped $\operatorname{In}_{0.05} \mathrm{Ga}_{0.95} \mathrm{~N}$ prelayer (identical to the prelayer contained in sample B). The multiple QW samples ${ }^{16}$ were found to be fully strained to the underlying GaN pseudo-substrate, suggesting that the SQW samples investigated here are also fully strained. Furthermore, previous studies have shown that no strain relaxation occurred in the case of $160 \mathrm{~nm}$ thick $\operatorname{In}_{0.05} \mathrm{Ga}_{0.95} \mathrm{~N}^{20}$ and $70 \mathrm{~nm}$ thick $\mathrm{In}_{0.12} \mathrm{Ga}_{0.88} \mathrm{~N}^{21}$ epilayers. Given that the prelayers in our samples are much thinner and contain less indium, we can reliably conclude that the inclusion of a $24 \mathrm{~nm}$ thick $\mathrm{In}_{0.05} \mathrm{Ga}_{0.95} \mathrm{~N}$ prelayer does not introduce strain relaxation in the structure.

The low temperature $(10 \mathrm{~K})$ PL spectra of each sample measured with an excitation power density of $15 \mathrm{Wcm}^{-2}$ are shown in Figure 1. The reference structure sample A has a peak emission energy of $2.635 \mathrm{eV}$, while samples $\mathrm{B}, \mathrm{C}$ and $\mathrm{D}$ have peak emission energies of $2.784 \mathrm{eV}$, $2.749 \mathrm{eV}$ and $2.756 \mathrm{eV}$ respectively. We attribute the emission at the peak energies quoted as being due to recombination of localized electrons and holes in the $\mathrm{QW}^{22,23}$. The features occurring $91 \mathrm{meV}$ lower in energy than the main PL peaks of each sample are due to longitudinal optical phonon accompanied recombination in the $\mathrm{QW}^{19}$. The additional emission peak at $2.921 \mathrm{eV}$ in the spectrum from sample $\mathrm{B}$ is also observed in a structure consisting of only an $\operatorname{In}_{0.05} \mathrm{Ga}_{0.95} \mathrm{~N}$ prelayer deposited on a GaN template and is thus attributed to carrier recombination within the prelayer itself. The peak emission energy from InGaN/GaN QWs is mainly determined by the thickness and In fraction of the QWs, as well as the magnitude of the Quantum Confined Stark Effect $^{1,24,25}$. As the thickness and In fractions of QWs 


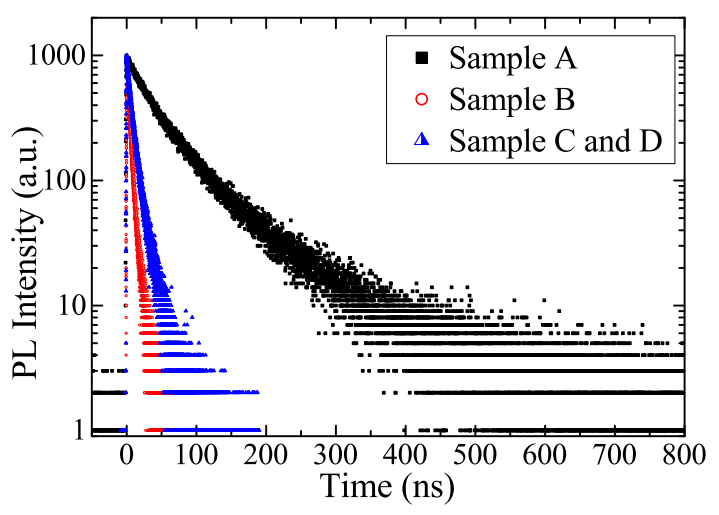

FIG. 2. PL decay transients measured at low temperature $(10 \mathrm{~K})$, at the peak emission energies of samples A, B, C and D. The PL decay transients of samples $\mathrm{C}$ and $\mathrm{D}$ are indistinguishable.

in each sample were measured to be the same, the spectroscopy suggests that the shift in the peak emission energy from samples B, C and D, compared with sample A, is due to a reduction of the QCSE. If this is the case we would expect a significant reduction in the radiative recombination lifetime. With this in mind, PL time decay measurements were performed on all four samples.

The low temperature $(10 \mathrm{~K})$ PL decay transients measured at the peak of the PL spectra for each sample are shown in Figure 2. As the intensities of the emission from the different samples were very similar we assume that the different decay curves reflect differences in the radiative recombination rates and not some differences in non-radiative recombination rates. In general the low temperature PL decay curves from InGaN/GaN QWs are non-exponential reflecting the effects of the variation in the wave function overlap of the separately localized electrons and holes ${ }^{22}$, and occur over a time scale which strongly reflects the strength of the in-built piezo- and polarization induced electric field ${ }^{19}$. For our measurements, as a means of characterizing the overall decay rates, we quote the time $\left(\tau_{10}\right)$ required for the PL intensity to drop by a factor 10 from its maximum value. The $\tau_{10}$ decay time measured for the reference sample $\mathrm{A}$ was $120 \mathrm{~ns} \pm 4 \mathrm{~ns}$, while the $\tau_{10}$ times for sample B, C and D were $9 \pm 1 \mathrm{~ns}, 19 \pm 1 \mathrm{~ns}$ and $19 \pm 1 \mathrm{~ns}$ respectively. At this point we note that the properties of the samples C and D that incorporate high-temperature and low-temperature grown GaN are essentially the same, so we conclude that the growth temperature of the prelayer does not influence the properties of the single QW but at the same time we stress that the presence of the doped GaN layer has a similar effect on the single QW to that of the doped InGaN. This tends to rule out any influence of a change in "V"-defects morphology occasioned by the prelayer on the single QW PL, since the surfaces on which the QWs are grown have identical morphology for the two samples grown at high temperature, A and D. (This point has been checked using relevant atomic force microscopy

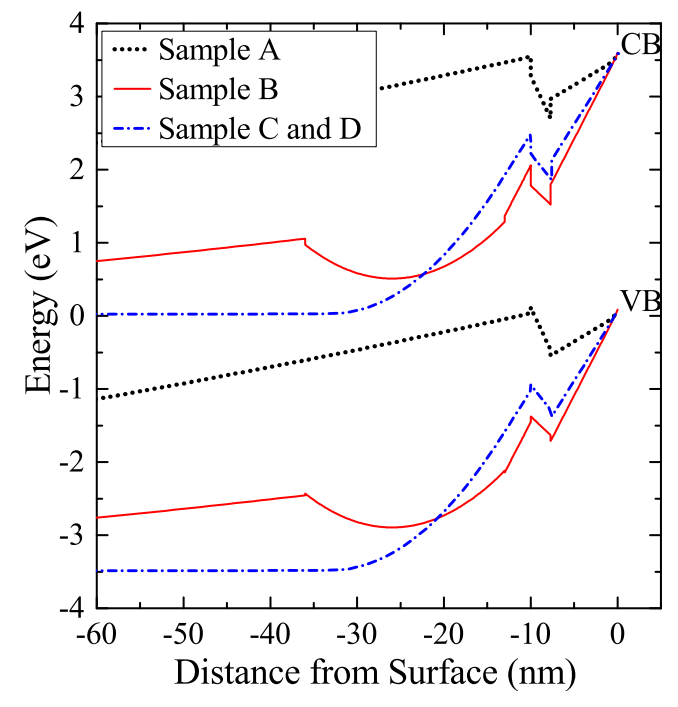

FIG. 3. Simulated profiles of the conduction (CB) and heavy hole valence (VB) bands for samples $\mathrm{A}, \mathrm{B}, \mathrm{C}$ and $\mathrm{D}$, shown as a function of depth from the sample surface. The profiles for samples $\mathrm{C}$ and $\mathrm{D}$ are indistinguishable as the only difference in these samples was the growth temperature of the GaN prelayers. A constant Fermi energy of $0 \mathrm{eV}$ was used in the simulation and has thus been used to define the $y$-axis in the figure.

studies).

The increases in PL peak emission energy and the radiative recombination rates as characterized by the $\tau_{10}$ values, for samples $\mathrm{B}, \mathrm{C}$ and $\mathrm{D}$ are consistent with a reduction in the strength of the electric field in the $\mathrm{QW}$, and hence a reduction in the QCSE. This is in line with a previous analyses of the effects of prelayers ${ }^{13,14,26}$ where the shift in the emission energies and the changes in the radiative decay rates were attributed to a change in the in-built electric field caused by strain relaxation, but in this case our X-ray measurements eliminate this as the reason for the effects of the prelayers. To understand the observed effects the conduction and valence band profiles in all the samples, shown in Figure 3, were calculated using a commercially available device simulator $\left(\text { nextnano }{ }^{3}\right)^{27}$.

The band profiles are largely determined by the changes in the position of the Fermi energy. Mayrock et $a l .{ }^{9}$ reported that due to a high density of unpaired bonds in Ga terminated GaN a large polarization charge exists at the sample surface, sufficient to pin the Fermi level at the valence band edge. However in $n$-doped material the Fermi level is pinned at the donor energy below the conduction band edge, thus resulting in a bending of the conduction and valence bands towards to the surface, as reported in $n$-type GaN epilayers ${ }^{9}$. The inclusion of the n-type doped regions (either Si-doped GaN or InGaN prelayers) therefore results in a strong modification of the band bending within the top $40 \mathrm{~nm}$ of the structure compared with sample A, shown in Figure 3. As the built-in potential gradient across the $\mathrm{QW}$, due to the combination 
of the spontaneous and piezoelectric polarizations, is in opposite sense to the potential gradient produced by the surface band bending, the resultant potential gradient across the QW in the prelayer samples $\left(111 \mathrm{meVnm}^{-1}\right.$ (for sample B) and $149 \mathrm{meVnm}^{-1}$ (for samples C and $\mathrm{D}))$ is much less than in sample A $\left(254 \mathrm{meVnm}^{-1}\right)$. This is consistent with the reduction in the QCSE and the increase in the radiative recombination rate. This broad agreement between the predictions of the modelling and the changes in the optical properties for the SQW structures that contain the prelayers goes beyond this initial comparison. We also note that the measured value of $\tau_{10}$ is smaller for sample $\mathrm{B}$ than sample $\mathrm{C}$ or $\mathrm{D}$, implying that the reduction in the QCSE is greater, which is in line with the predicted values for the potential gradients of $111 \mathrm{meVnm}^{1}$ and $149 \mathrm{meVnm}^{1}$ for samples with the InGaN prelayer and the GaN prelayers respectively. The presence of In within the prelayer of sample B forms an additional charged interface, of opposing sense to the bottom QW interface, due to the large polarisation discontinuity between $\mathrm{GaN}$ and $\mathrm{InGaN}{ }^{24}$. The close proximity of this additional charged interface to the $\mathrm{QW}$ in sample $\mathrm{B}(3 \mathrm{~nm})$ leads to a reduction in the resultant potential gradient across the QW, due to the partial cancellation of the opposing constituent charges.

In summary we have reported on a comparative study of SQW structures grown with and without prelayers. The prelayers were either a layer of GaN $23 \mathrm{~nm}$ thick Si-doped at $5 \times 10^{18} \mathrm{~cm}^{-3}$ or a $24 \mathrm{~nm}$ thick layer of $\mathrm{In}_{0.05} \mathrm{Ga}_{0.95} \mathrm{~N}$ also Si-doped at $5 \times 10^{18} \mathrm{~cm}^{-3}$. All of the single QW structures that incorporated the prelayers emitted light with a higher peak PL emission energy and a faster radiative recombination rate than the reference sample that did not include a prelayer. Simulations of the band profiles showed that the changes in PL properties of the prelayer samples occurred because of changes in the surface band bending caused by the doped prelayers, which in turn resulted in a reduction in electric field across the QW. The greatest effect was measured for the single QW deposited on the InGaN prelayer, which was explained by an additional smaller effect that occurs due to presence of an additional InGaN/GaN interface in close proximity to the QW and the partial cancellation of the electric fields from the oppositely charged interfaces. The effect of the doping of the prelayers was found to be more significant than the inclusion of indium within the prelayer. Clearly in this work we have shown how the optical properties of single QWs can be influenced by the presence of prelayers. Further work remains to be done to understand the role of prelayers on the properties of MQW structures and LEDs.

This work was carried out with the financial support of the United Kingdom Engineering and Physical Sciences Research Council under Grant Numbers EP/I012591/1 and $\mathrm{EP} / \mathrm{H} 011676 / 1$.
${ }^{1}$ T. Takeuchi, S. Sota, M. Katsuragawa, M. Komori, H. Takeuchi, H. Amano, and I. Akasaki, Japanese Journal of Applied Physics 36, L382 (1997).

${ }^{2}$ N. Otsuji, K. Fujiwara, and J. K. Sheu, J. Appl. Phys. 100, 113105 (2006).

${ }^{3}$ P. T. Törmä, O. Svensk, M. Ali, S. Suihkonen, M. Sopanen, M. A. Odnoblyudov, and V. E. Bougrov, J. Cryst. Growth 310, 5162 (2008).

${ }^{4}$ A. Hangleiter, F. Hitzel, C. Netzel, D. Fuhrmann, U. Rossow, G. Ade, and P. Hinze, Phys. Rev. Lett. 95, 127402 (2005).

${ }^{5}$ Q. Dai, M. F. Schubert, M. H. Kim, J. K. Kim, E. F. Schubert, D. D. Koleske, M. H. Crawford, S. R. Lee, A. J. Fischer, G. Thaler, and M. A. Banas, Appl. Phys. Lett. 94, 111109 (2009).

${ }^{6}$ A. Hangleiter, D. Fuhrmann, M. Grewe, F. Hitzel, G. Klewer, S. Lahmann, C. Netzel, N. Riedel, and U. Rossow, physica status solidi (a) 201, 2808 (2004).

${ }^{7}$ Y. L. Li, Y. R. Huang, and Y. H. Lai, Appl. Phys. Lett. 91, 181113 (2007).

${ }^{8}$ F. Bernardini, V. Fiorentini, and D. Vanderbilt, Phys. Rev. B 63, 193201 (2001).

${ }^{9}$ O. Mayrock, H.-J. Wünsche, and F. Henneberger, Phys. Rev. B 62, 16870 (2000).

${ }^{10}$ Y. Takahashi, A. Satake, K. Fujiwara, J. K. Shue, U. Jahn, H. Kostial, and H. T. Grahn, Physica E: Low-dimensional Systems and Nanostructures 21, 876 (2004).

${ }^{11}$ T. Akasaka, H. Gotoh, T. Saito, and T. Makimoto, Appl. Phys. Lett. 85, 3089 (2004).

${ }^{12}$ T. Akasaka, H. Gotoh, Y. Kobayashi, H. Nakano, and T. Makimoto, Appl. Phys. Lett. 89, 101110 (2006).

${ }^{13}$ N. Nanhui, W. Huaibing, L. Jianping, L. Naixin, X. Yanhui, H. Jun, D. Jun, and S. Guangdi, J. Cryst. Growth 286, 209 (2006).

${ }^{14}$ N. Nanhui, W. Huaibing, L. Jianping, L. Naixin, X. Yanhui, H. Jun, D. Jun, and S. Guangdi, Solid-State Electron. 51, 860 (2007).

${ }^{15}$ H. Takahashi, A. Ito, T. Tanaka, A. Watanabe, H. Ota, and K. Chikuma, Japanese Journal of Applied Physics 39, L569 (2000).

${ }^{16}$ M. J. Davies, F. C.-P. Massabuau, P. Dawson, R. A. Oliver, M. J. Kappers, and C. J. Humphreys, physica status solidi (c) 11, 710 (2014).

${ }^{17}$ S. J. Leem, Y. C. Shin, E. H. Kim, C. M. Kim, B. G. Lee, Y. Moon, I. H. Lee, and T. G. Kim, Semicond. Sci. Technol. 23, 125039 (2008).

${ }^{18}$ T. M. Smeeton, M. J. Kappers, J. S. Barnard, M. E. Vickers, and C. J. Humphreys, physica status solidi (b) 240, 297 (2003).

${ }^{19}$ D. M. Graham, A. Soltani-Vala, P. Dawson, M. J. Godfrey, T. M. Smeeton, J. S. Barnard, M. J. Kappers, C. J. Humphreys, and E. J. Thrush, J. Appl. Phys. 97, 103508 (2005).

${ }^{20}$ S. K. Rhode, W. Y. Fu, M. A. Moram, F. C.-P. Massabuau, M. J. Kappers, C. McAleese, F. Oehler, C. J. Humphreys, R. O. Dusane, and S. Sahonta, Appl. Phys. Lett. , (submitted) (2014).

${ }^{21}$ K. Pantzas, G. Patriarche, D. Troadec, S. Gautier, T. Moudakir, S. Suresh, L. Largeau, O. Mauguin, P. L. Voss, and A. Ougazzaden, Nanotechnology 23, 455707 (2012).

${ }^{22}$ A. Morel, P. Lefebvre, S. Kalliakos, T. Taliercio, T. Bretagnon, and B. Gil, Phys. Rev. B 68, 045331 (2003).

${ }^{23}$ P. G. Eliseev, M. Osinski, J. Lee, T. Sugahara, and S. Sakai, J. Electron. Mater. 29, 332 (2000).

${ }^{24}$ V. Fiorentini, F. Bernardini, F. Della Sala, A. Di Carlo, and P. Lugli, Phys. Rev. B 60, 8849 (1999).

${ }^{25}$ P. Lefebvre, A. Morel, M. Gallart, T. Taliercio, J. Allegre, B. Gil, H. Mathieu, B. Damilano, N. Grandjean, and J. Massies, Appl. Phys. Lett. 78, 1252 (2001).

${ }^{26}$ T. Li, Q. Y. Wei, A. M. Fischer, J. Y. Huang, Y. U. Huang, F. A. Ponce, J. P. Liu, Z. Lochner, J.-H. Ryou, and R. D. Dupuis, Appl. Phys. Lett. 102, 041115 (2013).

${ }^{27}$ nextnano ${ }^{3}$, "Semiconductor software solutions,". 